\title{
Intravitreal injection of plasminogen kringle 5, an endogenous angiogenic inhibitor, arrests retinal neovascularization in rats
}

\author{
D.Zhang, P. L. Kaufman, G. Gao, R. A.Saunders, J.X. Ma \\ Department of Ophthalmology, Medical University of South Carolina, Charleston, South Carolina, USA
}

\begin{abstract}
Aims/hypothesis. Plasminogen kringle 5 is an endogenous angiogenic inhibitor. The purpose of the present study was to explore the potential application of kringle 5 in the treatment of retinal neovascularization. Methods. Plasminogen kringle 5 was expressed in E. coli and affinity-purified. Its anti-angiogenic activity was determined in cultured primary human capillary endothelial cells. Retinal neovascularization was induced in newborn rats by exposure to hyperoxia and then normoxia. Kringle 5 was intravitreally injected into the rat model. Retinal neovascularization was visualized by fluorescein angiography on flat-mounted retina and quantified by counting preretinal vascular cells.

Results. Plasminogen kringle 5 inhibited primary endothelial cells but not retinal neuronal cells, suggesting cell type-specific inhibition. The oxygen-induced retinopathy rat model showed an over-expression of vascular endothelial growth factor, preretinal neovas-
\end{abstract}

cularization and haemorrhage. Intravitreal injection of kringle 5 before the development of neovascularization resulted in fewer neovascular tufts and pre-retinal vascular cells than in control rats with PBS injection $(p<0.01)$. Moreover, injection of kringle 5 after the development of neovascularization inhibited the increase in the preretinal vascular cells $(p<0.05)$. These results suggest that kringle 5 both prevents the development and arrests the progression of retinal neovascularization. The injection of kringle 5 did not result in any detectable inflammatory response in the retina or histological toxicity to retina neurons and pre-existing vessels.

Conclusion/interpretation. These observations suggest that intravitreal delivery of angiogenic inhibitors could have therapeutic benefits in neovascular diseases of the retina. [Diabetologia (2001) 44: 757-765]

Keywords Angiogenesis, angiogenic inhibitor, angiostatin, diabetes, retinopathy of prematurity.
Received: 2 October 2000 and in revised form: 27 December 2001

Corresponding author: Jian-xing Ma, MD, $\mathrm{PhD}$, Department of Ophthalmology, Medical University of South Carolina, 167 Ashley Ave, Charleston, SC 29403, USA

Abbreviations: bFGF, basic fibroblast growth factor; FBS, fetal bovine serum; HRCEC, human retinal capillary endothelial cell; IPTG, isopropylthio- $\beta$-galactoside; $\mathrm{K} 5$, kringle 5; PEDF, pigment epithelium derived factor; RGD, arginine-glycine-aspartic acid; ROP, retinopathy of prematurity; RT-PCR, reverse transcription-polymerase chain reaction; VEGF, vascular endothelial growth factor.
Retinal neovascularization, abnormal formation of new vessels from pre-existing capillaries, is a characteristic pathologic finding of many ocular diseases, such as diabetic retinopathy, sickle cell retinopathy, retinal vein occlusion and retinopathy of prematurity (ROP) [1, 2]. Ischaemia-induced neovascular retinopathies collectively comprise the most common cause of blindness and affect millions of people from infants to the elderly [3-5].

A common mechanism for neovascularization is the disturbed regulation of angiogenesis [6]. In normal ocular tissues, angiogenesis is regulated by two counter-balancing systems, angiogenic stimulators e.g. vascular endothelial growth factor, (VEGF) and 
angiogenic inhibitors $[1,7,8]$. It is hypothesized that under certain pathological conditions such as diabetic retinopathy and ROP, the retina increases the expression of angiogenic factors while decreasing the production of angiogenic inhibitors in response to local hypoxia. As a result, capillary endothelial cells proliferate excessively, leading to retinal neovascularization $[1,8]$.

Plasminogen kringle 5 (K5) is a proteolytic fragment of plasminogen $[9,10]$. Plasminogen contains 5 kringle domains; each kringle consists of 80 amino acids [11]. Kringle 5 has been shown to inhibit proliferation of endothelial cells. Moreover, its inhibitory activity is more potent than angiostatin (i.e. kringles $1-4)[9,10,12]$. In addition, $\mathrm{K} 5$ also inhibits endothelial cell migration, an important process in angiogenesis $[13,14]$. It exerts its effect on endothelial cells by inducing cell cycle arrest and apoptosis and is specific to proliferating endothelial cells [14]. Because of its high efficacy, cell type-selectivity, and short amino acid sequence, $\mathrm{K} 5$ has considerable potential in the treatment of neovascular diseases $[10,13]$. In the present study, we examined the in vitro and in vivo effects of recombinant $\mathrm{K} 5$ on retinal neovascularization.

\section{Subjects and methods}

Animals. Sprague Dawley and Brown Norway rats were purchased from Harlan (Indianapolis, Ind., USA). Care, use, and treatment of all animals in this study were in strict agreement with the ARVO Statement for the Use of Animals in Ophthalmic and Vision Research, as well as the guidelines set forth in the Care and Use of Laboratory Animals by the Medical University of South Carolina.

Construction of K5 expression vector. The human K5 cDNA was amplified from liver RNA by reverse transcription-polymerase chain reaction (RT-PCR) as described previously [15]. The 5' PCR primer (5'-TGTGAATTCGCCAGATGTAGAGACTCCTTC-3') and the 3 ' primer (5'-GGAAAGCTTGGCACACTGAGGGACATCACAG-3') contain Eco RI and Hind III sites, respectively, to facilitate cloning. The PCR product was cloned into the pET22 vector (Novagen, Madison, Wis., USA) at the Eco RI and Hind III sites in frame with the signal peptide sequence at the $5^{\prime}$ end and the sequence encoding $6 \mathrm{x}$ His at its $3^{\prime}$ end. The K5/pET22 construct was sequenced to ensure PCR fidelity and correct reading frame.

Expression and purification of K5 in E. coli. The K5/pET22 construct was introduced into E. coli strain BL-21/DE3 (Novagen, Madison, Wis., USA). This vector provides a signal peptide that enables the recombinant protein to enter the periplasmic space. The expression and purification followed the protocol recommended by Novagen with some modifications. Briefly, expression was induced by the addition of isopropylthio- $\beta$ galactoside (IPTG) and carried out for $10 \mathrm{~h}$ at $25^{\circ} \mathrm{C}$. Periplasmic proteins were released by digestion with lysozyme and separated from cells by centrifugation. K5 was purified by passing through the His-Bind column (Novagen). The purity and identity of recombinant K5 was examined by SDS-PAGE and
Western blot analysis using an antibody specific to His-tag (Oncogene Research Products, Cambridge, MA) or anti-K5 antibody (a generous gift from Dr. H. R. Lijnen, Center for Molecular and Vascular Biology, University of Leuven, Belgium) as described previously [15].

Isolation and culture of human retinal capillary endothelial cells. Human retinal capillary endothelial cells (HRCEC) were isolated from donor eyes, obtained through the South Carolina Lion's Eye Bank Association, as described previously [16]. At the second passage, the purity of endothelial cells in culture was confirmed by incorporation of acetylated low density lipoprotein labelled with the fluorescent probe, DiI (1,1'dioctadecyl-3,3,3',3'-tetramethylindocarbocyanine perchlorate, Biomedical Technologies, Stoughton, Mass., USA) [16].

Viable cell count. Cells between the second and sixth passages were seeded in gelatin/fibronectin-coated 12 -well plates at a density of $104 /$ well and cultured in a medium containing $15 \%$ FBS until the cells reached $50-70 \%$ confluency. The medium was then changed to one containing $5 \%$ FBS and $1 \mathrm{ng} / \mathrm{ml}$ bFGF. We added K5 to the above medium at different concentrations in quadruplicate. The cells were then cultured under normoxic or hypoxic $\left(1 \% \mathrm{O}_{2}\right)$ conditions for $72 \mathrm{~h}$. Cells were detached from the wells by trypsinization, resuspended in growth media, and stained with $0.4 \%$ trypan blue. Viable cells were then counted under light microscope. Average cell number of each K5 treated group was individually compared to that of the control group using the Student's $t$ test. The differences were considered significant if $p$ was less than 0.05 .

Induction of retinal neovascularization and quantification of preretinal nuclei. Induction and quantification of retinal neovascularization were performed as described previously $[17,18]$ with some modifications. Briefly, newborn Sprague Dawley or Brown Norway rats were randomly assigned to each experimental group. The rats at postnatal day 7 (P7) were exposed to hyperoxia $\left(75 \% \mathrm{O}_{2}\right)$ for 5 days and then normoxia for another 7 days to induce retinal neovascularization. For comparison of the sensitivity of different rat strains to the oxygen-induced retinopathy, a total of 24 Sprague Dawley and 24 Brown Norway rats were randomly assigned into two groups, 12 animals each were maintained in the normoxia as controls and the other 12 were subjected to the oxygen treatment. Of the 12 animals in each group, the right eyes of 8 rats were sectioned for quantification of pre-retinal vascular cells and the left eyes were pooled for Western blot analysis of VEGF concentrations. The other 4 animals were used for fluorescein angiography. For quantification of preretinal nuclei, eyes were fixed with $10 \%$ formaldehyde, sectioned and then stained with hematoxylin and eosin. The nuclei of vascular cells on the vitreal side of the retina were counted using light microscopy $(\times 250)$. A total of 8 sagittal sections from each eye were examined and cell numbers were averaged in each group of animals $[17,18]$. The average number of pre-retinal nuclei was compared to the control group by Student's $t$ test.

Retinal angiography with high molecular weight fluorescein. Altogether 4 animals per group were anaesthetized and perfused with fluorescein by an intra-ventricle injection of $50 \mathrm{mg}$ / $\mathrm{ml}$ of high molecular weight $\left(2 \times 10^{6}\right)$ fluorescein isothiocyanate-dextran (Sigma) as described previously [17]. The animals were immediately killed, and the eyes were enucleated and fixed in $4 \%$ paraformaldehyde for $10 \mathrm{~min}$. The retina was dissected free of the lens and vitreous and incubated in $4 \%$ paraformaldehyde for $3 \mathrm{~h}$. The retina was cut and flat-mount- 


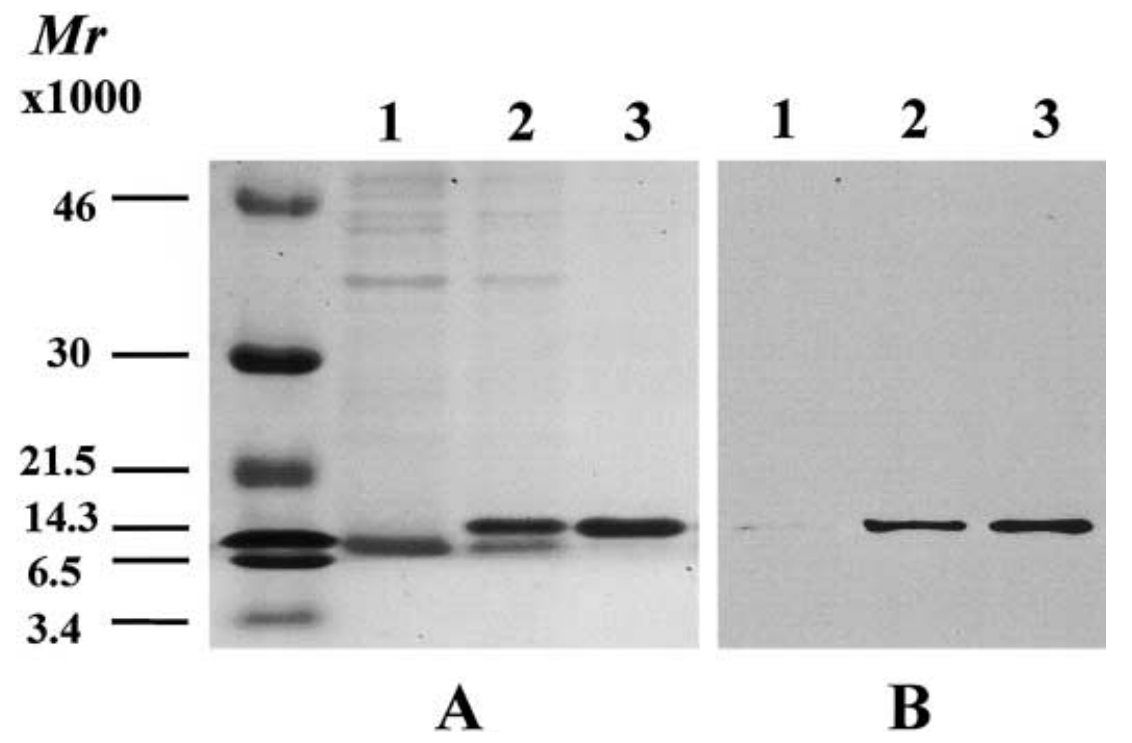

Fig.1. Expression and purification of recombinant K5: Panel A, SDS-PAGE with Coomassie blue staining; $\mathbf{B}$, western blot analysis with antibody specific to K5. Lane 1, crude periplasmic protein before the IPTG induction; 2, crude periplasmic protein after the IPTG induction and 3, affinity-purified K5

ed on a gelatin-coated slide. The vasculature was then examined under a fluorescent microscope (Axioplan2 Imaging, Zeiss, Thomwood, N. Y., USA).

Western blot analysis. The retina was dissected, pooled within a group and homogenized by sonication. The protein concentration in the supernatant was measured with the BioRad protein assay. One hundred $\mu \mathrm{g}$ of soluble protein were resolved by SDS-PAGE (15\% polyacrylamide gel) and electro-transferred to Hybond ECL nitrocellulose membrane (Amersham Pharmacia Biotech, Piscataway, N. Y., USA). The membrane was then blocked with $5 \%$ BLOTTO solution in TBST buffer (20 mmol/l Tris-Cl, pH 7.6, $137 \mathrm{mmol} / \mathrm{l} \mathrm{NaCl}, 0.1 \%$ Tween 20 ) with shaking for $1 \mathrm{~h}$ at room temperature. The rabbit antiVEGF antibody (Santa Cruz Biotechnology Santa Cruz, Calif., USA) was added to the BLOTTO to 1:4000 and incubated with the membrane overnight at $4{ }^{\circ} \mathrm{C}$ with shaking. Each membrane was washed three times with TBST at room temperature, 20 min. A donkey anti-rabbit antibody labelled with horseradish peroxidase (Amersham International) was diluted in the BLOTTO solution to 1:4000 and incubated with the membrane for $2 \mathrm{~h}$ at room temperature. The membrane was washed three times with TBST. The band was visualized by ECL detection kit (Amersham International) and exposed to a Kodak X-Omat film. The same membrane was stripped and re-blotted with an antibody specific to $\beta$-actin.

Intravitreal injection of K5. Recombinant K5 was dissolved in PBS and sterilized by filtration. It was injected either immediately after the animals were returned from hyperoxia to normoxia or at 7 days after the return. The animals were anaesthetized and $\mathrm{K} 5(0.3$ and $3 \mu \mathrm{g} / \mu \mathrm{l})$ was injected into the vitreous $(3 \mu \mathrm{l} / \mathrm{eye})$ of the right eye through the pars plana using a glass capillary needle. The left eye received the same volume of PBS and was used as the control. For each group, 12 Brown Norway rats were used, 4 for fluorescein angiography and 8 for quantification of preretinal nuclei on sections. After injection, the animals were kept in normoxia for 7 days and then killed.

\section{Results}

Expression and purification of K5. After the IPTG induction of the expression, the periplasmic fraction was separated from the cell and K5 was purified to apparent homogeneity with the His.Bind affinity column. The recombinant protein showed an apparent molecular weight of $16 \mathrm{kDa}$, matching the calculated molecular weight based on the sequence (Fig.1A). The identity of the recombinant K5 was confirmed by western blot analysis using anti-His tag and antiK5 antibodies (Fig.1B). An average of $10-20 \mathrm{mg}$ of purified $\mathrm{K} 5$ was obtained from the periplasmic fraction of 11 of culture. Endotoxin concentrations in the purified protein were below $0.00625 \mathrm{EU} / \mathrm{ml}$ as measured using a limulus amebocyte kit (Biowittaker, Helsinki, Finland).

In vitro inhibition of HRCEC by recombinant $K 5$ under normoxic and hypoxic conditions. The HRCEC were treated with recombinant $\mathrm{K} 5$ at concentrations of $20,40,80,160 \mathrm{nmol} / 1$ under normoxic or hypoxic $(1 \% \mathrm{O} 2)$ conditions for $72 \mathrm{~h}$ and subjected to cell count. We found that HRCEC under the hypoxic condition showed more viable cells than in normoxia $(p<0.05, n=4)$ (Fig. 2). At a concentration as low as $20 \mathrm{nmol} / \mathrm{l}, \mathrm{K} 5$ reduced cell numbers $(p<0.01, n=4)$ under both the normoxic and hypoxic conditions (Fig. 2). As shown in Figure 2, the inhibitory activity appeared to be K5 concentration-dependent. The apparent $\mathrm{EC}_{50}$ was $70 \mathrm{nmol} / \mathrm{l}$ under normoxia and 60 nmol/l under hypoxia.

Under the same conditions, K5 did not show any inhibition of E1A-NR3 cells ( $p>0.05, n=4)$, an im- 


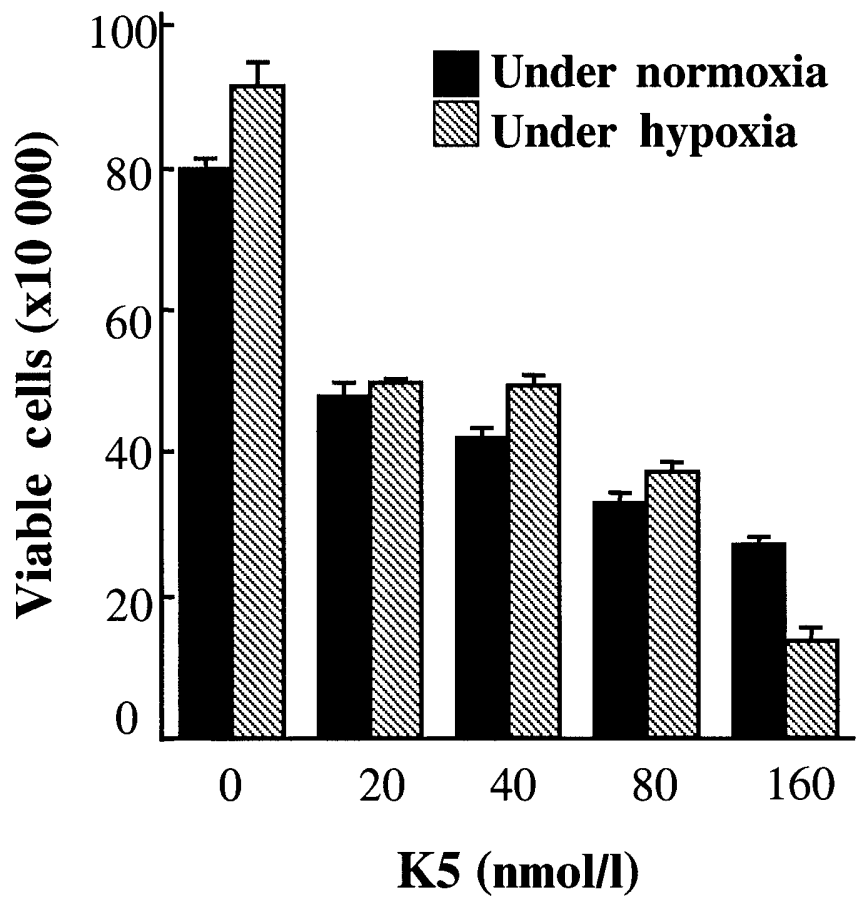

Fig. 2. Effect of K5 on HRCEC: HRCEC were treated with recombinant $\mathrm{K} 5$ at the concentrations as indicated under the normoxic and hypoxic conditions. The cells were treated for $72 \mathrm{~h}$ and viable cells counted. Bars represent means \pm SEM $(n=4)$

mortalized cell line manifesting phenotypes of retinal photoreceptor cells [19]. This lack of effect on the retinal cells was observed at K5 concentrations from 20-320 nmol/l, suggesting that K5's inhibition is specific to vascular endothelial cells.

Inhibitory activity independent of RGD. Kringle 5 contains a sequence homologous to the RGD motif, which is known to interfere with integrin binding. In order to determine whether the inhibitory activity of $\mathrm{K} 5$ on HRCEC is mediated by the RGD motif, the RGDV peptide (Advanced Chemtech, Louisville, Ky., USA) was applied to cultured HRCEC under the same conditions used for K5. At concentrations from $1-500 \mu \mathrm{mol} / \mathrm{l}$, the RGDV peptide did not show any inhibition of HRCEC growth $(p>0.05, n=4)$, suggesting that the inhibitory activity of $\mathrm{K} 5$ is independent of the RGD motif.

Oxygen-induced retinopathy in Brown Norway rats. Retinal neovascularization was induced by exposure of newborn Brown Norway rats to hyperoxia for 5 days and then to normoxia [17]. At 7 days after the return to the normoxia, retinal examination revealed significant retinal neovascularization, microaneurysms, enlarged non-perfusion regions, and haemorrhage in all of the Brown Norway rats examined (Fig.3-4). Western blot analysis showed that VEGF concentrations were significantly induced in the reti- na by the oxygen treatment, after normalization with $\beta$-actin concentrations (Fig. 3D). The induced VEGF expression is consistent with the previous observation in the mouse model [20]. Retinal neovascularization was quantified by counting preretinal nuclei in saggital sections, as previous studies showed that the number of pre-retinal nuclei were correlated with retinal neovascularization [17, 21]. Quantification of pre-retinal neovascular cells showed that Brown Norway rats developed more neovascular cells than albino Sprague Dawley rats under the same conditions $(p<0.01, n=8)$ (Fig.4E), suggesting that Brown Norway rats are more sensitive than Sprague Dawley rats to oxygen-induced retinopathy. Therefore, all subsequent experiments were performed using Brown Norway rats.

K5 prevents the development of retinal neovascularization in vivo. In order to determine its preventive effect, $\mathrm{K} 5$ was injected into the vitreous immediately after the return of the animals from hyperoxia to normoxia, as our study showed that at this time point neovascularization had not yet occurred (data not shown). The rats were then kept in normoxia for 7 days, wherein retinal vasculature was examined. Eyes that received $3 \mu \mathrm{l}$ of $3 \mu \mathrm{g} / \mu \mathrm{l} \mathrm{K5}$ injection showed a reduction in neovascular tufts as compared to control eyes receiving the same volume of PBS (Fig.5). The K5 treated eyes showed fewer preretinal neovascular cells than the control eyes, treated with PBS $(33.3 \pm 2.7$ nuclei/section in the K5-treated vs $66.1 \pm 5.9$ in PBS-treated, $p<0.01, n=8$ ) (Fig. 5F). This result suggests that a single $\mathrm{K} 5$ injection at least partially prevents the development of retinal neovascularization under ischaemic conditions. Injection of the same volume of $0.3 \mu \mathrm{g} / \mu \mathrm{l} \mathrm{K} 5$ did not result in significant reduction in neovascular cells $(65.3 \pm 2.0 \mathrm{nu}-$ clei/section, $p=0.9, n=8$ ), suggesting that the inhibitory effect is dependent on the $\mathrm{K} 5$ concentration.

K5 arrests the progression of retinal neovascularization in vivo. In order to determine the effect of $\mathrm{K} 5$ after the development of retinal neovascularization, K5 was injected intravitreally at 7 days after returning the rats to normoxia, wherein obvious retinal neovascularization had occurred (Fig. 3). The rats were kept for an additional 7 days in normoxia to allow the progression of the retinal neovascularization to occur and then the neovascular cells quantified. In K5treated eyes, the mean neovascular cell number increased slightly from $74.6 \pm 12.4$ nuclei per section before the injection to $80.3 \pm 22.5(p=0.4, n=8)$ 7 days after the injection (Fig. 6). In contrast, the neovascular cell number in the control group with PBS injection increased almost twofold, from $74.6 \pm 12.4$ nuclei per section to $145.5 \pm 24.1(p<0.01, n=8)$. Statistical analysis demonstrates that the eyes treated with K5 had fewer neovascular cells than the PBS-in- 

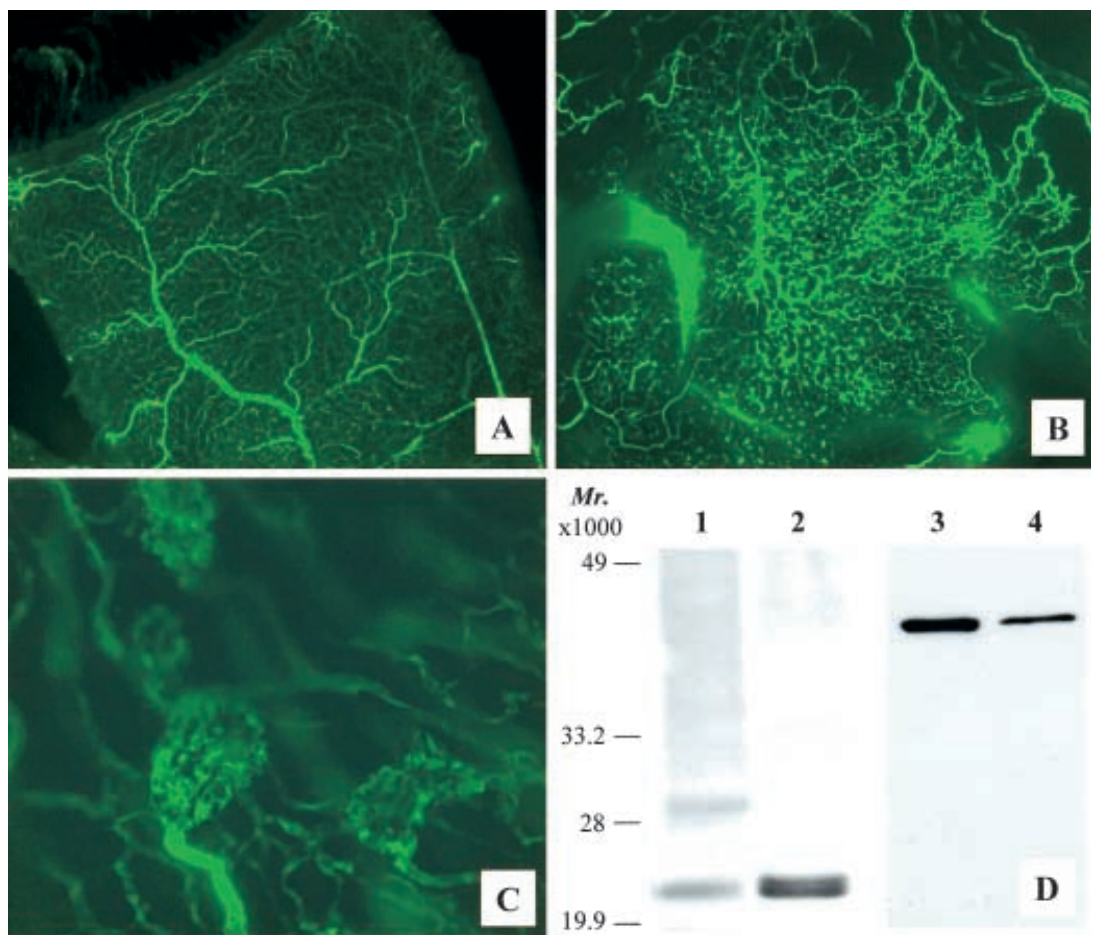

\section{Mr.}

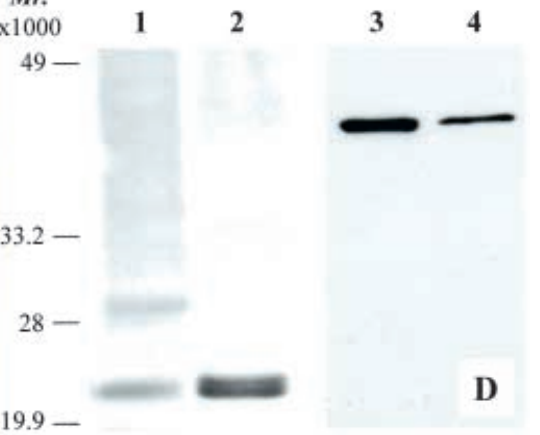

Fig.3. Oxygen-induced retinopathy in rats: Newborn Brown Norway rats were exposed to hyperoxia for 5 days and then normoxia for another 7 days. Retinal neovascularization was analyzed by fluorescein-dextran angiography and western blot analysis of VEGF. A, normal control animals kept in normoxia $(\times 50) ; \mathbf{B}$ and $\mathbf{C}$, oxygen-induced retinopathy $(\mathrm{B}, \times 50$ and $\mathrm{D}, \times 400) ; \mathrm{D}$, western blot analysis of VEGF (lanes $1 \& 2$ ) and $\beta$-actin (lanes $3 \& 4$ ); lanes $1 \& 3$, normal controls; $2 \& 4$, oxygen-treated rats

jected control group at 7 days after the injection $(p<0.05, \mathrm{n}=8)$ (Fig. 6). However, the $\mathrm{K} 5$ injection did not decrease the number of pre-existing vascular cells (Fig. 6). These results suggest that a single K5 injection arrests the progression of neovascularization but does not affect pre-existing vascular endothelial cells.

Structure of the retina after injection of K5. To determine the possible toxicity and inflammatory responses resulting from $\mathrm{K} 5$ injection, retinal structure was examined 7 days after the injection. No apparent histological change or inflammatory cells infiltrate was observed in the retinas in any of the examined sections (Fig. 5). In addition, there was no detectable change in the normal intraretinal vasculature after injection of $\mathrm{K} 5$. The finding suggests that $\mathrm{K} 5$, at the concentrations used, does not cause detectable toxicity to the retina or normal vasculature.

\section{Discussion}

Retinal neovascularization is a major cause of vision loss in human beings. Currently, laser photocoagulation and, in some instances, cryotherapy are used to treat neovascularization in the posterior segment $[22,23]$. Although these therapies generally improve vision, they are not ideal modes of treatment, because they can be ineffective and are often associated with substantial morbidity, such as nyctalopia and the loss of peripheral vision [1, 22, 23]. More effective prevention or treatment strategies are therefore desirable [1, 24].

The potential therapeutic effect of angiogenic inhibitors such as angiostatin and endostatin in the treatment of cancer has been studied extensively [12, 25, 26]. However, their application in the treatment of ocular neovascular diseases, such as ROP and diabetic retinopathy, has not been explored. In the present study, we demonstrate that a single intravitreal injection of recombinant plasminogen $\mathrm{K} 5$ both prevents the development and arrests the progression of retinal neovascularization in rats. This inhibitory activity appears to be specific for proliferating endothelial cells and is not associated with detectable retinal toxicity. This study represents the first approach to treating retinal neovascularization using an endogenous angiogenic inhibitor.

Investigators have long sought a satisfactory in vivo model of retinal neovascularization. Studies in the 1970 s revealed that most diabetic rat and mouse models do not develop typical retinal neovascularization even after long periods of hyperglycaemia $[1,27]$. Investigators have $[17,18]$ developed an oxy- 

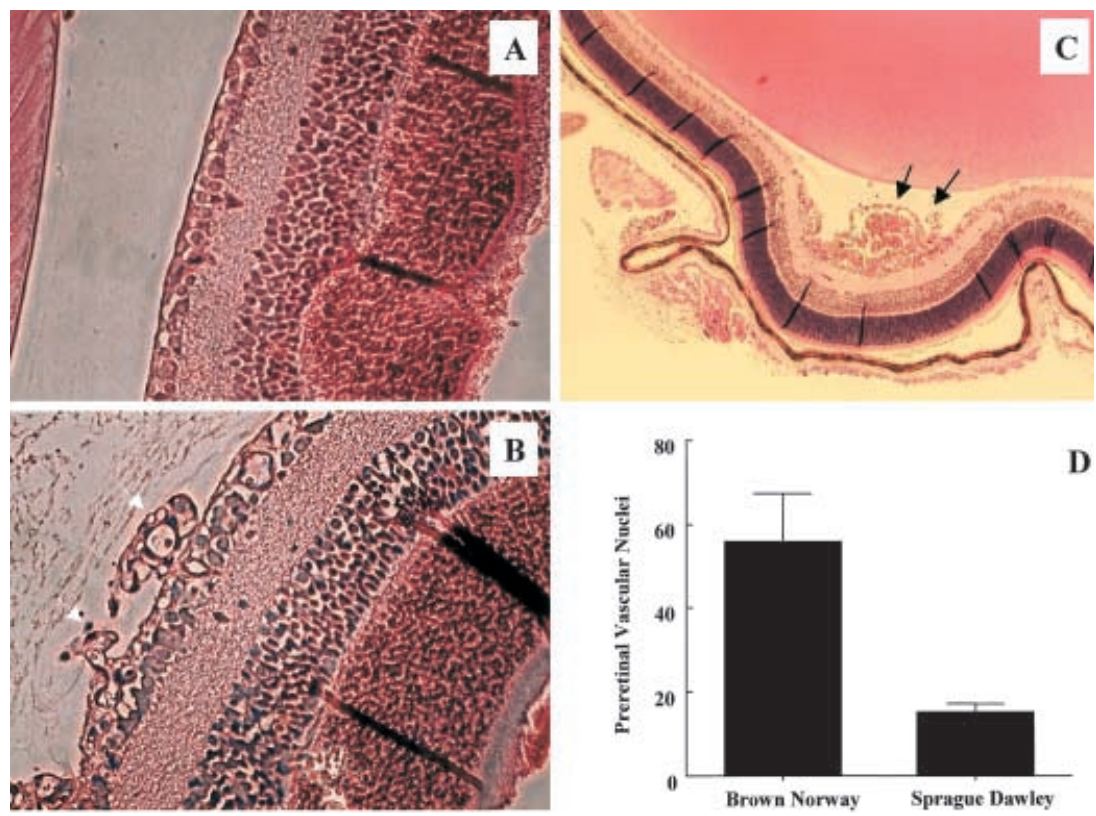

Fig.4. Quantification of oxygen-induced neovascular cells: Newborn animals were exposed to 5 days of hyperoxia and then to normoxia for 7 days. Pre-retinal vascular cells were quantified on saggital sections. A, normal control $(\times 400)$; $\mathbf{B}$ and C, oxygen-treated Brown Norway rats $(\times 400$ and $\times 100$, respectively); $\mathbf{D}$, average numbers of pre-retinal vascular cells in Brown Norway and Sprague Dawley rats (means \pm SEM, $n=8$ )

gen-induced retinopathy model in newborn mouse. The mechanism by which neovascularization occurs in this model is similar to that of the ischaemic retinopathies such as ROP and diabetic retinopathy $[1$, 20]. Thus, the oxygen-induced retinopathy appears to be a suitable animal model for the study of similar retinal conditions in humans [17, 18, 20]. Unlike mice, it has been shown that it is more difficult to induce retinal neovascularization in newborn Sprague Dawley rats $[28,29]$. Instead of exposure to constant hyperoxia followed by normoxia, the Sprague Dawley rats require cycling between hyperoxia and normoxia to induce neovascularization $[28,29]$. Indeed, our results using the Sprague Dawley rats support these findings. In contrast, Brown Norway rats developed more severe retinal neovascularization after exposure to constant hyperoxia followed by normoxia. This finding suggests strain differences with regard to their responses to oxygen-induced retinopathy. As such, pigmented Brown Norway rats appear to be a more suitable model for oxygen-induced retinopathy using the protocol previously described [17].

Since the disturbed balance between angiogenic stimulators and inhibitors is the common mechanism leading to retinal neovascularization, we hypothesize that delivery of a potent angiogenic inhibitor or a combination of several inhibitors should prevent or arrest the process of neovascularization. Several anti-angiogenic approaches have been tested previously in the oxygen-induced retinopathy model [21, $30,31]$. Although a number of synthetic angiogenic inhibitors are available, they are usually associated with unacceptable toxicity and adverse effects [32-34]. Systemic administration of cyclic peptide RGDfK has been shown to reduce retinal neovascularization without affecting established vessels or visible side effects $[30,35]$. However, local administration of angiogenic inhibitors is more desirable since a long-term systemic administration of angiogenic inhibitors is more likely to cause adverse effects on physiologic angiogenesis involved in such events as wound healing, and thus, is not suitable for patients with diabetes or ROP.

Other investigators [21, 31] have convincingly demonstrated that blocking VEGF expression or activity by intravitreal delivery of antisense oligonucleotides of VEGF and soluble VEGF-neutralizing chimeric proteins inhibits retinal neovascularization. These pioneer studies demonstrated the potential of intravitreal anti-angiogenic therapy in the treatment of retinal neovascularization. In addition to VEGF, however, other angiogenic stimulators could also contribute to retinal neovascularization [6]. In an effort to block the final pathway of angiogenesis rather than targeting a single growth factor, our study aims to employ endogenous angiogenic inhibitors, such as angiostatin and $\mathrm{K} 5$, as they can counter-balance the angiogenesis induced by multiple factors $[10,12]$. Although a number of endogenous angiogenic inhibitors (e.g. angiostatin, K5, PEDF, endostatin) have been identified in the past several years and are widely believed to have therapeutic potential for neovascular diseases $[8,10,12,25,36]$, their applications in 

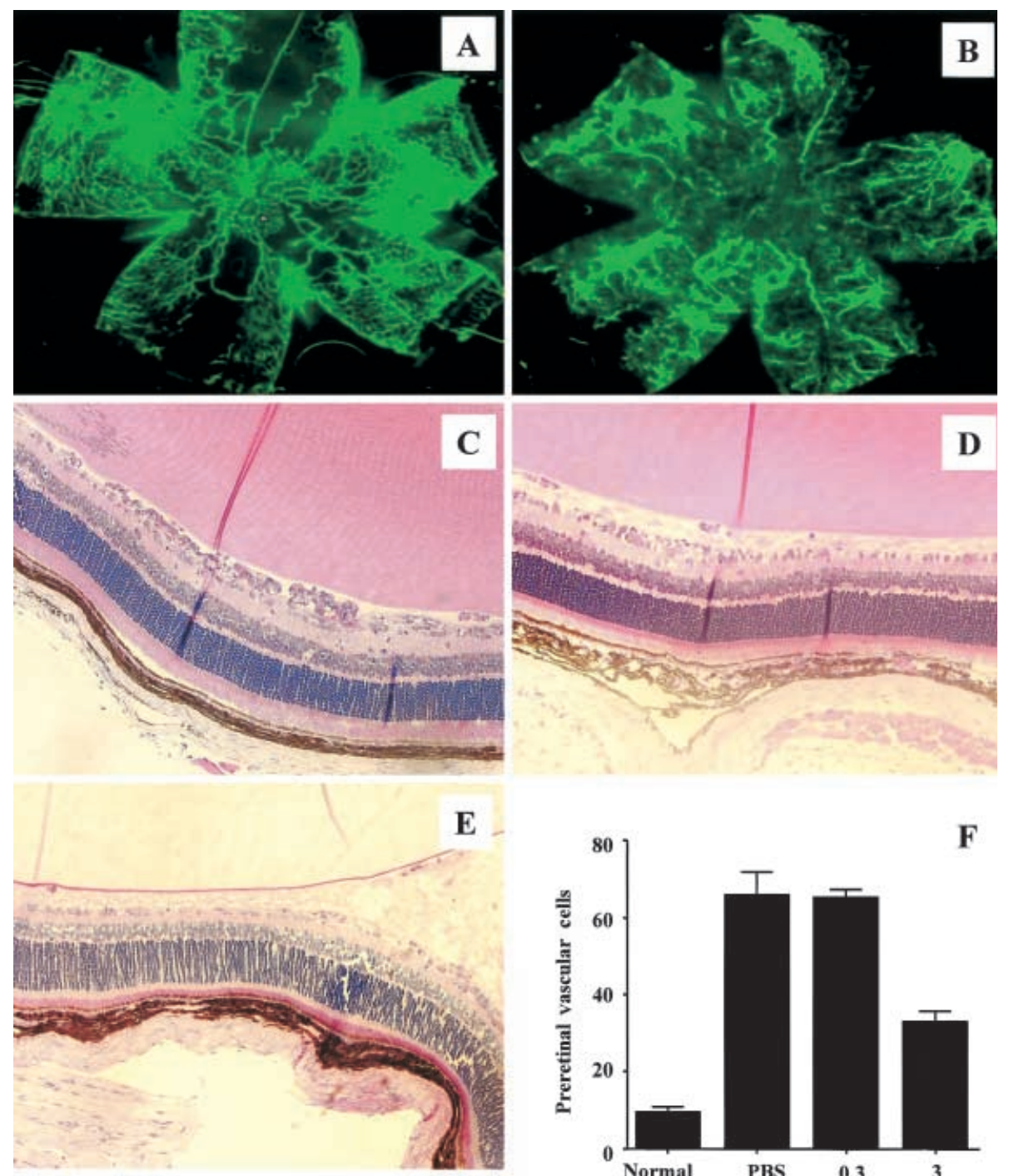

$\mathbf{E}$

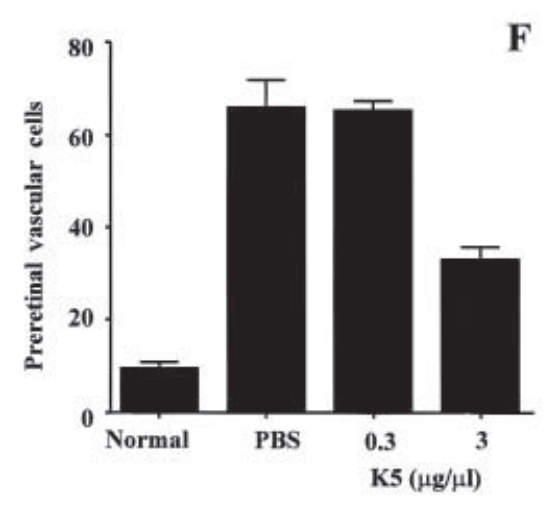

Fig.5. Effect of K5 on the development of retinal neovascularization: K5 was injected immediately after the animals were returned to normoxia from hyperoxia. The animals were kept in normoxia for another 7 days and retinal neovascularization was examined. A, Whole-mounted retina of oxygen-treated eyes with PBS injection and $\mathbf{B}$, with K5 injection $(\times 12.5)$; C, retinal section from oxygen-treated rat with PBS injection; D, oxygen-treated rat with $\mathrm{K} 5$ injection; E, control rats kept in normoxia $(\times 200)$ and $\mathbf{F}$, pre-retinal vascular cell numbers (means \pm SEM) in the groups treated with K5 and PBS (control) $(n=8)$

the treatment of retinal neovascularization have not yet been explored.

In the present study, we chose $\mathrm{K} 5$ as the first candidate for anti-angiogenesis therapy because of the following perceived advantages. Firstly, plasminogen is endogenously expressed in ocular tissues (unpublished data), and therefore, $\mathrm{K} 5$ is not likely to induce an immune response. Secondly, K5 exerts its inhibito-

ry activity specific to proliferating vascular endothelial cells and has low toxicity to non-proliferating endothelial cells and other cell types [10,14]. Thirdly, K5 is a small peptide and easy to produce in E. coli. Finally, K5 is relatively stable and, thus, suitable for a sustained-releasing delivery system. Our results confirm that a single intravitreal injection of K5 both prevents retinal neovascularization and arrests its progression without detectable toxicity in the animal model. The data therefore suggest that endogenous peptide $\mathrm{K} 5$ is promising as a potential therapeutic drug for retinal neovascularization.

Although evidence demonstrates that K5 induces apoptosis and inhibits proliferation in proliferating endothelial cells, the molecular mechanism is not known at the present time [14]. It is known that K5 contains a sequence motif homologous to the RGD peptide. The RGD peptide is known to be the integrin-binding site responsible for cell adhesion [37]. After coating a surface, RGD promotes cell adhesion. When RGD is in solution, however, it prevents 


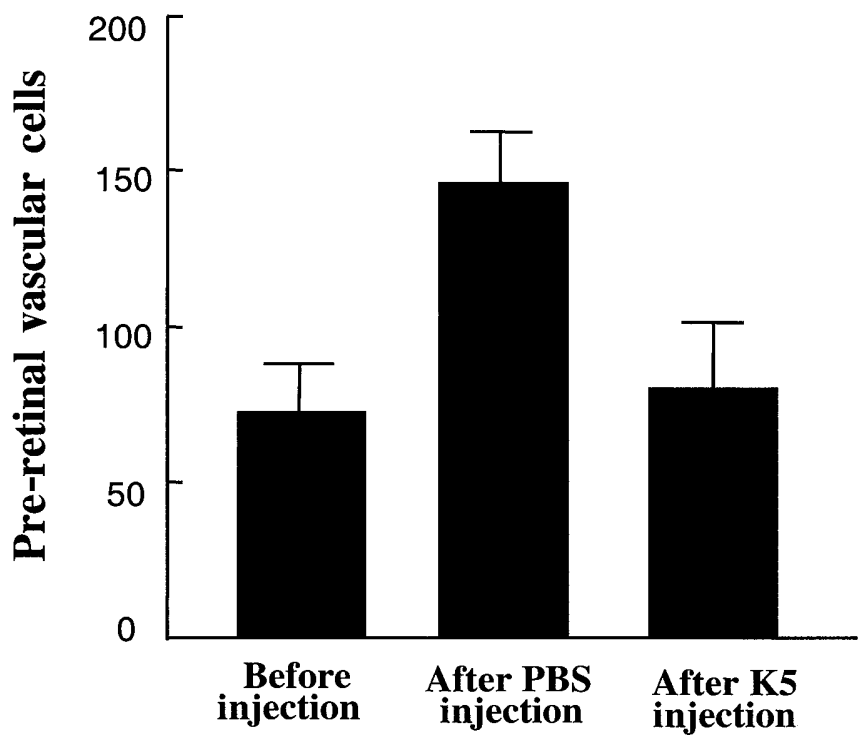

Fig.6. Effect of $\mathrm{K} 5$ on the progression of retinal neovascularization: Animals were exposed to hyperoxia for 5 days, followed by 7 days of normoxia. Animals received a single injection of K5 or PBS and were then kept for another 7 days in normoxia to allow the progression of retinal neovascularization. Vascular cells in the vitreous were counted and expressed as means \pm SEM in the groups of rats before the injection, 7 days after PBS injection, and 7 days after the K5 injection $(n=8)$

endothelial cell adhesion, with consequent anti-angiogenic activity [37]. In order to determine whether the anti-angiogenic activity of $\mathrm{K} 5$ is mediated by the RGD motif, RGD peptide was applied to primary HRCEC. At concentrations up to 1000 -fold higher than that of K5, RGD peptide did not show any significant inhibition of HRCEC, suggesting that the inhibitory activity of $\mathrm{K} 5$ is achieved through a yet to be identified mechanism independent of the anti-adhesion effect of the RGD motif. This result is consistent with observations from angiostatin studies [38].

The present study demonstrates that an angiogenic inhibitor can be effectively administered not only before the development of neovascularization but also after retinal neovascularization has occurred. Thus, this local anti-angiogenic therapy can be applied not only to prevent retinal neovascularization but also to stop its progression, once established. Moreover, K5 blocks the progression of retinal neovascularization but does not reduce the number of pre-existing vascular cells. This result suggests that K5, similar to angiostatin [39], affects only proliferating endothelial cells but not pre-existing normal endothelial cells. This apparent specificity will be an important feature for a clinically useful anti-angiogenic drug. These observations further suggest that endogenous angiogenic inhibitors have great potential in the treatment of retinal neovascularization in human beings.

It is noteworthy that the preventive effect of a single dose of K5 is only partial, possibly due to the rap- idly decreasing concentration of $\mathrm{K} 5$ in the vitreous. Our preliminary studies show that the $t_{1 / 2}$ of $K 5$ in the vitreous is less than 2 days after a single injection (unpublished data). Improving the availability of K5 in the vitreous remains a future challenge, because treatment with repeated intravitreal injections is not desirable. The development of novel delivery techniques, such as a sustained-releasing pellet or gel, or gene delivery, will be necessary to achieve prolonged availability of an angiogenic inhibitor in the vitreous after a single dose injection [40, 41]. If improved methods of delivery can be developed, intravitreal injection of natural angiogenic inhibitors with regulated releasing rates could become an effective therapy for preventing the development or inhibiting the progression of retinal neovascularization in prethreshold to threshold ROP and in the pre-proliferative to early stages of proliferative diabetic retinopathy.

Acknowledgements. The authors would like to thank Drs. M. Buse and R. K. Crouch at the Medical University of South Carolina for critical review of this manuscript, Dr. H. R. Lijnen, at the Center for Molecular and Vascular Biology, University of Leuven, Belgium, for the generous gift of antibody specific to K5 and Dr. G. Seigel at Rochester University for E1A-NR3 cells. This work was supported by NIH grant EY12600 and EY12231, a Research Grant from Juvenile Diabetes Association and an unrestricted grant to Department of Ophthalmology, MUSC from Research to Prevent Blindness.

\section{References}

1. Miller J (1997) Vascular endothelial growth factor and ocular neovascularization. Am J Pathol 151: 13-23

2. Steinkuller PG, Du L, Gilbert C, Foster A, Collins ML, Coats DK (1999) Childhood Blindness. J AAPOS 3: 26-32

3. Klein R, Klein BE, Moss SE (1992) Epidemiology of proliferative diabetic retinopathy. Diabetes Care 15: 1875-1891

4. Blom ML, Green WR, Schachat AP (1994) Diabetic retinopathy: a review. Del Med J 66: 379-388

5. Thylefors B, Negrel AD, Pararajasegaram R, Dadzie KY (1995) Global data on blindness. Bull World Health Organ 73: $115-121$

6. Robinson GS, Aiello LP (1998) Angiogenic factors in diabetic ocular disease: mechanisms of today, therapies for tomorrow. Int Ophthalmol Clin 38: 89-102

7. Bussolino F, Mantovani A, Persico G (1997) Molecular mechanisms of blood vessel formation. Trends Biochem Sci 22: 251-256

8. Dawson DW, Volpert OV, Gillis P et al. (1999) Pigment epithelium-derived factor a potent inhibitor of angiogenesis. Science 285: 245-248

9. Cao Y, Ji RW, Davidson D et al. (1996) Kringle domains of human angiostatin. Characterization of the anti-proliferative activity on endothelial cells. J Biol Chem 271: 29461-29467

10. Cao Y, Chen A, An SSA et al. (1997) Kringle 5 of plasminogen is a novel inhibitor of endothelial cell growth. J Biol Chem 272: 22924-22928

11. Castellino FJ, McCance SG (1997) The kringle domains of human plasminogen. Ciba Found Symp 212: 46-65 
12. O'Reilly MS, Holmgren L, Shing Y et al. (1994) Angiostatin: a novel angiogenesis inhibitor that mediates the suppression of metastases by a Lewis Lung Carcinoma. Cell 79: $315-328$

13. Ji W-R, Barrientos LG, Llinas M et al. (1998) Selective inhibitor by kringle 5 of human plasminogen on endothelial cell migration, an important process in angiogenesis. Biochem Biophys Res Commun 247: 414-419

14. Lu H, Dhanabal M, Volk R et al. (1999) Kringle 5 causes cell cycle arrest and apoptosis of endothelial cells. Biochem Biophy Res Commun 285: 668-673

15. Ma J-X, Zhang D, Laser M et al. (1999) Identification of RPE65 mRNA in transformed kidney cells. FEBS Lett 452: 199-204

16. Grant MB, Guay C (1991) Plasminogen activator production by human retinal endothelial cells of non-diabetic and diabetic origin. Invest Ophthalmol Vis Sci 32: 53-64

17. Smith LE, Wesolowski E, McLellan A et al. (1994) Oxygen-induced retinopathy in the mouse. Invest Ophthalmol Vis Sci 35: 101-111

18. Smith LEH, Kopchick JJ, Chen W et al. (1997) Essential role of growth hormone in ischemia-induced retinal neovascularization. Science 276: 1706-1709

19. Seigel GM (1996) Establishment of an E1A-immortalized retinal cell culture. In Vitro Cell Dev Biol Anin 32: 66-68

20. Pierce EA, Avery RL, Foley ED, Aiello LP, Smith LEH (1995) Vascular endothelial growth factor/vascular permeability factor expression in a mouse model of retinal neovascularization. Proc Natl Acad Sci USA 92: 905-909

21. Aiello LP, Pierce EA, Foley ED et al. (1995) Suppression of retinal neovascularization in vivo by inhibition of vascular endothelial growth factor (VEGF) using soluble VEGF-receptor chimeric proteins. Proc Natl Acad Sci USA 92: 10457-10461

22. Wilkes SR (1993) Current Therapy of diabetic retinopathy: laser and vitreoretinal surgery. J Natl Med Assoc 85: 841-847

23. Regillo CD, Brown GC, Flynn HW Jr (1999) Vitreoretinal Disease: The Essentials. Thieme Medical Publishers, New York

24. Battegay EJ (1995) Angiogenesis: mechanistic insights, neovascular diseases, and therapeutic prospects. J Mol Med 73: 333-346

25. O'Reilly MS, Boeham T, Shing Y et al. (1997) Endostatin: an endogenous inhibitor of angiogenesis and tumor growth. Cell 88: 277-285

26. Boehm T, Folkman J, Browder T, O’Reilly MS (1997) Antiangiogenic therapy of experimental cancer does not induce acquired drug resistance. Nature 390: 404-407
27. Engerman RL (1976) Animal models of diabetic retinopathy. Trans Am Acad Ophthalmol Otolaryngol 81: 710-715

28. Penn JS, Tolman BL, Henry MM (1994) Oxygen-induced retinopathy in the rat: relationship of retinal nonperfusion to subsequent neovascularization. Invest Ophthalmol Vis Sci 35: 3429-3435

29. Reynaud X, Dorey CK (1994) Extraretinal neovascularization induced by hypoxic episodes in the neonatal rat. Invest Ophthalmol Vis Sci 35: 3169-3177

30. Hammes H-P, Brownlee M, Jonczyk A, Sutter A, Preissner KT (1996) Subcutaneous injection of a cyclic peptide antagonist of vitronectin receptor-type integrins inhibits retinal neovascularization. Nat Med 2: 529-533

31. Robinson GS, Pierce EA, Rook SL, Foley E, Webb R, Smith LEH (1996) Oligodeoxynucleotides inhibit retinal neovascularization in a murine model of proliferative retinopathy. Proc Natl Acad Sci USA 93: 4851-4856

32. D'Amato RJ, Loughnan MS, Flynn E, Folkman J (1994) Thalidomide is an inhibitor of angiogenesis. Proc Natl Acad Sci USA 91: 4082-4085

33. Kato T, Sato K, Kakinuma H, Matsuda Y (1994) Enhanced suppression of tumor growth by combination of angiogenesis inhibitor. Cancer Res 54: 5143-5147

34. Folkman J, Ingber D (1992) Inhibition of angiogenesis. Semin Cancer Biol 3: 89-96

35. Friedlander M, Theesfeld CL, Sugita M et al. (1996) Involvement of integrins avb3 and avb5 in ocular neovascular diseases. Proc Natl Acad Sci USA 93: 9764-9769

36. O'Reilly MS, Pirie-Shepherd S, Lane WS, Folkman J (1999) Antiangiogenic activity of the cleaved conformation of the serpin antithrombin. Science 285: 1926-1928

37. Ruoslahti E (1996) RGD and other recognition sequences for integrins. Ann Rev Cell Dev Biol 12: 697-715

38. Claesson-Welsh L, Welsh M, Ito N et al. (1998) Angiostatin induces endothelial cell apoptosis and activation of focal adhesion kinase independently of the integrin-binding motif RGD. Proc Natl Acad Sci USA 95: 5579-5583

39. Luo J, Lin J, Paranya G, Bischoff J (1998) Angiostatin upregulates E-selectin in Proliferating endothelial cells. Biochem Biophys Res Commun 245: 906-911

40. Ozaki H, Hayashi H, Vinores SA, Moromizato Y, Campochiaro PA, Oshima K (1997) Intravitreal sustained release of VEGF causes retinal neovascularization in rabbits and breakdown of the blood retinal barrier in rabbits and primates. Exp Eye Res 64: 505-517

41. Hsu HT, Wong CG, Rich KA, Bonakdar M, Lee C (1999) Model of proliferative diabetic retinopathy: retinal neovascularization induced by intravitreal sustained release of VEGF and bFGF in the pigmented rabbit. Invest Ophthalmol Vis Sci 40: S704 (Abstract) 